\title{
OPTIMIZATION OF THE SHX FUSION POWERED TRANSATMOSPHERIC PROPULSION CONCEPT
}

\author{
Robert B. Adams" \\ National Aeronautics and Space Administration \\ Marshall Space Flight Center \\ MSFC, AL 35812 \\ D. Brian Landrum' \\ University of Alabama in Huntsville \\ Propulsion Research Center \\ Tech Hall, S234 \\ Huntsville, Alabama 35899
}

\begin{abstract}
Existing propulsion technology has not achieved cost effective payload delivery rates to low earth orbit. A fusion based propulsion system, denoted as the Simultaneous Heating and eXpansion (SHX) engine, has been proposed in earlier papers. The SHX couples energy generated by a fusion reactor to the engine flowpath by use of coherent beam emitters. A quasi-one-dimensional flow model was used to quantify the effects of area expansion and energy input on propulsive efficiency for several beam models. Entropy calculations were included to evaluate the lost work in the system.
\end{abstract}

\section{INTRODUCTION}

Space exploration in the $21^{\text {st }}$ century will require cost efficient propulsion systems for propelling payloads into earth orbit. The argument can be made that this requirement should be the primary goal for propulsion researchers for the near future. Approaches to reducing launch costs typically involve increasing engine efficiency. Increasing efficiency allows more payload to be carried to orbit. It also allows the inclusion of additional systems to increase redundancy (and thereby increase safety) and to reduce operations requirements.

This paper describes the Simultaneous Heating and eXpansion jet (SHXjet) concept. The SHXjet system uses a coherent energy beam, such as a laser or electron gun, to deposit energy into the working air. A fusion reactor generates the energy in order to avoid the complications of fission reactors. The energy deposition acts to thermally choke the airflow. Therefore the engine flowpath is designed with a changing axial area profile to alleviate thermal choking. The result is an engine flow that receives large amounts of power from a compact energy source. The flow experiences few obstructions and can be expanded while heating to produce a high energy exhaust. The resultant thrust may be suitable for launch applications either as primary propulsion or for thrust augmentation.

\footnotetext{
Systems Engineer, member AIAA.

'Associate Professor, Department of Mechanical and Aerospace Engineering, senior member AIAA.
}

There are several potential advantages that the SHXjet offers over traditional airbreathing systems. The small compact fuel source eliminates the need for large, heavy fuel tanks typical of current systems. A reduced tankage requirement may produce smaller vehicles, thereby realizing an aerodynamic advantage. The SHXjet may potentially operate over a greater Mach number range than traditional airbreathing engines. Should this prove true, the SHXjet could replace several airbreathing engine cycles, thereby simplifying the overall engine system.

A full analysis of all the potential advantages would require simulation of the SHXjet performance for several vehicle concepts through a full trajectory. Such an endeavor was considered beyond the scope of this study. This project attempts to determine whether the SHXjet can produce similar performance to a conventional scramjet at a point in a typical flight trajectory of a combined cycle propelled launch vehicle. Towards this end, the authors created a computer model to determine the gasdynamic properties of the SHXjet propulsion flowpath from the vehicle tip to tail. The simulation also models the mass and power specifications of the fusion and beam emitter subsystems. A complementary simulation of a scramjet engine was performed for purposes of comparison.

It should be noted that there is not a practical fusion reactor in operation today. The SHXjet concept anticipates the difficulties with maintaining a fusion reaction will be overcome and a viable, flight capable fusion system will eventually be deployed. Until that 
happens, the research described here can help address the systems engineering issues of using a fusion source in a highly efficient earth to orbit vehicle.

\section{SHX SIMULATION MODEL}

\section{Overview}

The SHXjet model simulates the gasdynamics of the engine flowpath from the vehicle tip to its tail. The model also calculates engine performance, weight and power requirements for the fusion power and beam emitter subsystems. Figure 1 is a schematic of a theoretical SHXjet powered vehicle. The vehicle flowfield can be divided into several regions, each involving different underlying phenomena. Stations are defined with numeric designations for the interfaces between the flowpath components consistent with those defined in Heiser and Pratt'. Each of the regions illustrated in the figure are described in the following sections.

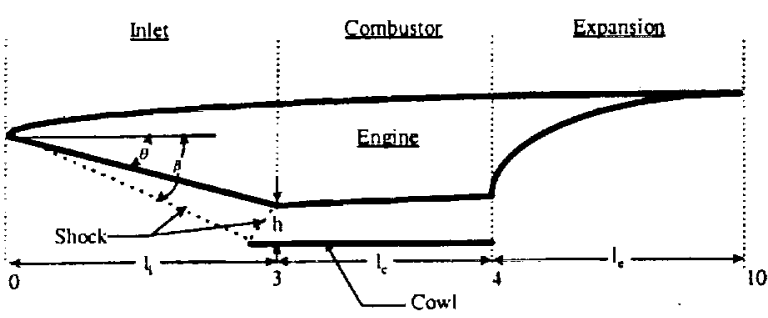

Figure 1. Diagram of a baseline vehicle geometry

\section{Fusion Reactor}

There are several concepts currently being researched that have the potential to achieve practical fusion in the near future. One promising concept is the Quiet Electric Discharge (QED) system ${ }^{2}$, which uses inertial electrostatic confinement to achieve fusion. Figure 2 illustrates an expanded view of the fusion chamber. A negative potential well is created in the center of the fusion chamber through the injection of electron beams. The well is restrained by an externally applied electric field, which is created using concentric grids inside the fusion chamber. The charged particles resulting from the fusion reaction expand against the electric field. The interaction between the charged particles and the electric field creates a voltage differential that can be used for power production. The bottom of Figure 2 illustrates the voltage differential between the collector shell and the ion well.

Bussard ${ }^{2}$ developed several empirical equations that predict the weight of a QED fusion engine. These relations are included in the model. The QED rocket concept uses an electron beam to heat the fuel. The current SHXjet concept uses lasers or an electron gun assembly. It is assumed that the laser assembly will have a similar weight to the e-beam assembly for a given power output.

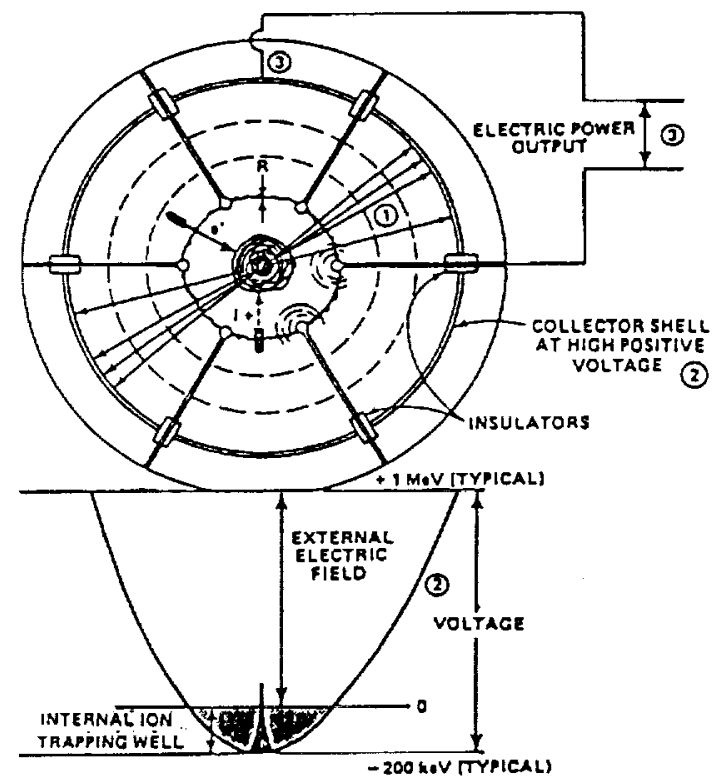

Figure 2. Fusion chamber and electrical conversion diagram for the QED system ${ }^{2}$

\section{Concept Summary}

Figure 3 illustrates the power flow through three SHX engine concepts. In all cases the fusion reactor produces the necessary power, $P_{\text {fision }}$. The conversion between the electrical energy generated by the reactor to beam energy is assumed to be $50 \%$. At this point the SHX operation deviates based on the type of beam emitter.

In the HF laser option (Figure 7.a) the power from the reactor is used to disassociate the HF reactants, which cycle between the laser and a disassociation chamber. The disassociated reactants then flow into the lasing cavity. The laser beam is then directed into the engine flowpath.

In the $\mathrm{CO}_{2}$ laser mode (Figure 7.b) the fusion power is used to drive the electrical discharge across the lasing cavity. Again the conversion efficiency is assumed to be $50 \%$. The fuel power, the product of the mass flow rate and the heating value $\left(\mathrm{HV}_{\mathrm{H} 2}\right)$ of hydrogen, is shown coming out of the $\mathrm{H}_{2}$ tank. The amount of power that actually makes it into the flowpath is the product of the fuel power, the mixing efficiency and the combustion efficiency. In this study the beam power for the $\mathrm{CO}_{2}$ laser is reduced by the fuel power amount in order to maintain the same total power as the other beam emitter options.

For the e-beam emitter option (Figure 7.c) the ebeam generator converts the electrical power from the reactor into a coherent beam with a $50 \%$ efficiency. The beam is then directed into the flow. 


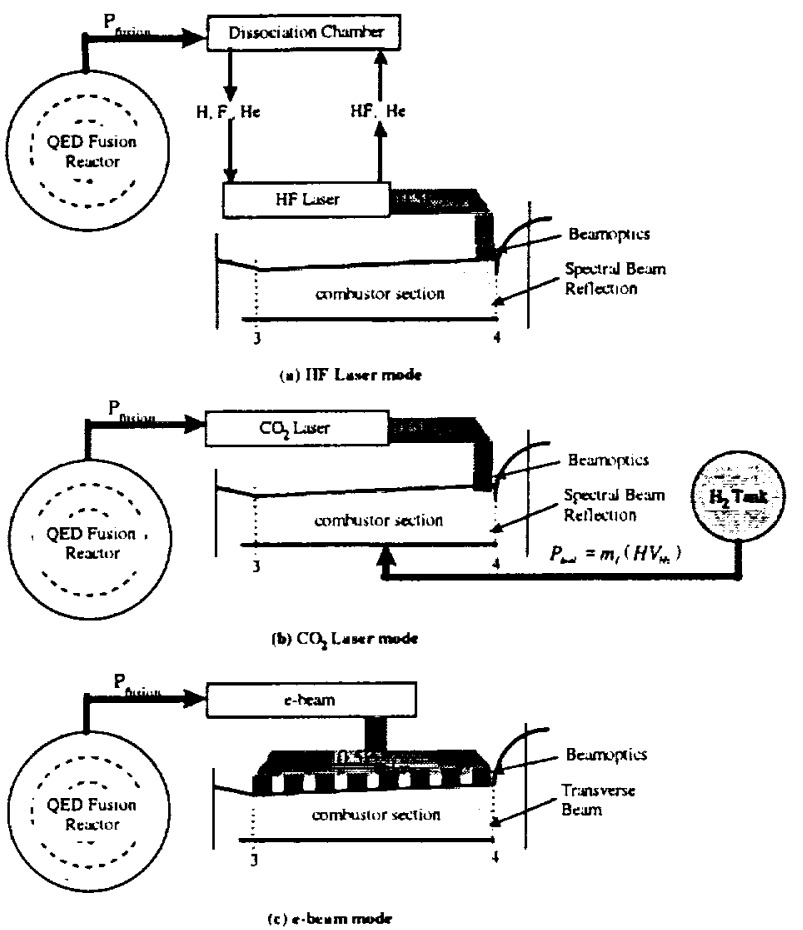

Figure 3. Power flow through the SHX engine

\section{ENTROPY CALCULATIONS}

A couple of modifications to the SHX code were made as part of $t$ his project. Entropy calculations were added in order to evaluate the efficiency of the heating process. Initial entropy was estimated at the inlet to the expansion section based on the inlet temperature and pressure and assuming standard air. The change in entropy was calculated at each axial position using

$$
\Delta s=c_{p} \ln \frac{T_{2}}{T_{1}}-R \ln \frac{p_{2}}{p_{1}},
$$

where subscripts 1 and 2 refer to the conditions at the previous and current node, respectively. The equilibrium calculations reevaluate $c_{p}$ and $R$ at each node while the frozen calculations use the averaged values from the equilibrium run. Note that the axial entropy plots should be evaluated by their change from the inlet value as absolute entropy values depend on the defined reference state.

\section{OVERALL EFFICIENCY}

The overall efficiency is defined as the amount of thrust power generated from the engine divided by the total power output from the reactor. It is shown in equation form as

$$
\eta_{s}=\frac{F V_{0}}{P_{\text {reurtur }}},
$$

Due to the power conversion factor assumed in the beam emitter subsystem the total efficiency for these runs can never exceed $50 \%$. This parameter will indicate the effectiveness of the given engine configuration in converting the allotted power to thrust.

\section{RESULTS}

This paper addresses the sensitivity of engine performance to the engine area configuration and beam power. Analyses on engine performance have been made using the code described in references 3 and 4 .

The first analysis set involves the use of the e-beam subsystem. The e-beam emitter assumes a linear heating profile across the length of the combustor section and thus is the simplest. The cases, shown in Table 1, are designed to consider ${ }^{-}$the effect of combustor expansion and energy input on overall engine performance. The inlet geometry and freestream conditions are the same for all of the cases. Also the overall expansion from the combustor inlet to the expansion discharge is held constant. In this manner the flow from the inlet experiences the same overall expansion regardless of proportion of expansion in the combustor section. The similar constants for these cases are shown in Table 2.

The points in Table 1 are designed to calculate a reasonable range of performance for a given expansion ratio. There are two restraints used to determine the range. The first is that the flow cannot be expanded to the point where the exit pressure is near the zero point. At this point the code becomes unstable and does not converge. This is a 'soft' limit, i.e. there was no attempt to find the points of lowest heat input that did not invoke this condition. The second restraint is that of thermal choking. The code can only calculate flows that are supersonic throughout the axial length. Past a certain point the energy input will 'choke' the flow and force it into a subsonic condition. This is a 'hard' limit, i.e. the analysis found the highest energy input (within $500 \mathrm{MW}$ ) that did not force a thermal choke.

Figure 4 illustrates the variation in specific thrust with energy input for several combustor expansion ratios. The reported specific thrusts approach and exceed that of typical scramjets $(80-100 \mathrm{lbf}-\mathrm{sec} / \mathrm{bm})$ at the higher energy inputs. Several trends are evident in this figure. First, the specific thrust is higher at the lower expansion ratios for a given energy input. Second, the specific thrust varies roughly linearly with energy input for a given expansion ratio. That is intuitive as the more energy that is input into the flow the more is available for conversion to thrust. Third, as the expansion ratio increases the more energy is required to force a thermal choke. Again, that makes sense as the higher expansions in the engine section counteract the decelerating effects of the energy inputs.

Fourth, the frozen assumption generates higher calculated performances than that using the equilibrium assumption. In fact the difference in performance 
increases steadily with increasing energy input. This too is intuitive, as the higher energy inputs would be expected to yield higher gas temperatures that would activate vibrational and electronic modes in the air molecules. Activation of such modes requires energy that would not then be available for conversion to thrust.

Table 1. e-beam cases

\begin{tabular}{|c|c|c|c|}
\hline \multirow[b]{2}{*}{ Case } & \multicolumn{2}{|c|}{ Area Ratio } & \multirow{2}{*}{$\begin{array}{l}\text { Heat } \\
\text { Input }\end{array}$} \\
\hline & Combustor & Expansion & \\
\hline I & 2.0 & 9.0 & 1500 \\
\hline 2 & 2.0 & 9.0 & 2600 \\
\hline 3 & 2.5 & 7.2 & 1500 \\
\hline 4 & 2.5 & 7.2 & 2000 \\
\hline 5 & 2.5 & 7.2 & 2500 \\
\hline 6 & 2.5 & 7.2 & 3000 \\
\hline 7 & 2.5 & 7.2 & 3500 \\
\hline 8 & 3.0 & 6.0 & 3000 \\
\hline 9 & 3.0 & 6.0 & 3500 \\
\hline 10 & 3.0 & 6.0 & 4000 \\
\hline 11 & 3.0 & 6.0 & 4500 \\
\hline 12 & 3.0 & 6.0 & 5000 \\
\hline 13 & 3.5 & 5.14 & 2000 \\
\hline 14 & 3.5 & 5.14 & 2500 \\
\hline 15 & 3.5 & 5.14 & 3000 \\
\hline 16 & 3.5 & 5.14 & 3500 \\
\hline 17 & 3.5 & 5.14 & 4000 \\
\hline 18 & 3.5 & 5.14 & 4500 \\
\hline 19 & 3.5 & 5.14 & 5000 \\
\hline 20 & 3.5 & 5.14 & 5500 \\
\hline 21 & 3.5 & 5.14 & 6000 \\
\hline 22 & 4.0 & 4.5 & 1000 \\
\hline 23 & 4.0 & 4.5 & 2000 \\
\hline 24 & 4.0 & 4.5 & 3000 \\
\hline 25 & 4.0 & 4.5 & 4000 \\
\hline 26 & 4.0 & 4.5 & 5100 \\
\hline 27 & 4.0 & 4.5 & 5500 \\
\hline 28 & 4.0 & 4.5 & 6500 \\
\hline
\end{tabular}

Calculation of the engine thrust in the chart above involves the use of the stream thrust function

$$
S a=u\left[1+\frac{R T}{u^{2}}\right] \text {, }
$$

The stream thrust function is used to calculate the net thrust as follows

$$
F=\dot{m}\left[S a_{10}-S a_{0}+\frac{R T_{0}}{u_{0}}\left(\frac{A_{10}}{A_{0}}-1\right)\right],
$$

where subscripts 0 and 10 indicate the inlet and discharge of the engine respectively. Since the inlet configuration for all cases are the same the inlet stream thrust function does not change. Also, the mass flow rate is the same for all of the cases. Although the combustor area ratio is varied the expansion area ratio is adjusted for each case to keep the total area ratio Thus the difference in thrust between cases is a function

Table 2. Standard variables for SHX code

of the differences in exit temperature and velocity.

\begin{tabular}{ll}
\hline \multicolumn{1}{c}{ Variable } & Value \\
\hline Mach number & 5.0 \\
Altitude & $65750 \mathrm{ft}$ \\
Bow angle & $25 \mathrm{deg}$ \\
Inlet type & Two-shock internal-external \\
Combustor inlet height & $3.0 \mathrm{ft}$ \\
Combustor inlet width & $12.0 \mathrm{ft}$ \\
Combustor length & $40.0 \mathrm{ft}$ \\
Expansion length & $50.0 \mathrm{ft}$ \\
Area profile & Parabolic \\
\hline
\end{tabular}

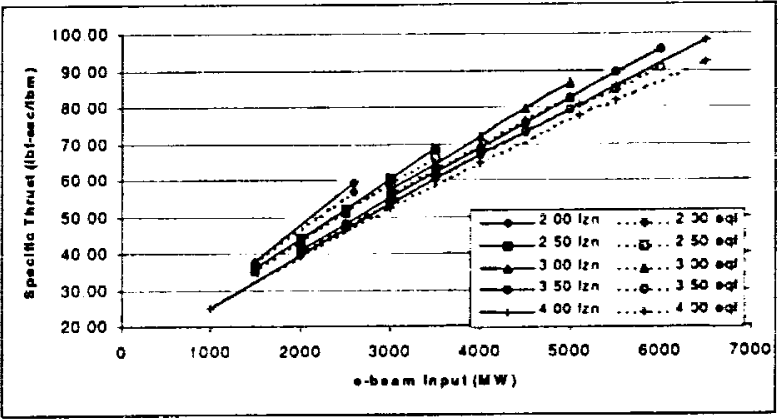

Figure 4. Specific thrust vs. e-beam energy input

The values given above do not account for the possibility of shock structures in the expansion section to equilibrate the discharge pressure with the ambient pressure. This was neglected on purpose in order to observe trends without the added variable of whether there were nozzle shocks. In this manner the values in Figure 4 can be considered 'pseudo-vacuum' specific thrusts even though a vacuum thrust could not be produced with a true airbreathing engine. The cases at $3000 \mathrm{MW}$ input and lower produce exit pressures lower than the freestream pressure and would have a shock structure in the expansion section in a true setting. The pressure losses across the shock would reduce the engine thrust and thus the specific thrust.

Figure 5 illustrates the change in the thrust to engine weight ratio with increasing energy input and changing combustor area ratios. Immediately obvious is the inverse relationship between thrust to weight ratio and energy input. The shapes of the curves suggest that thrust to weight is proportional to the heat input to a negative power. This relationship is depicted by 


$$
\frac{T}{W} \propto \frac{1}{Q^{n}},
$$

A less pronounced effect is the decrease in thrust to weight ratio with increasing combustor area ratios. A typical scramjet operates in a thrust to engine weight ratio of 7-10 therefore the cases illustrated here meet or exceeds that of a comparable scramjet. Again the equilibrium thrust to weight values lag those calculated by the frozen assumption. The difference in values is more pronounced at the higher energy input rates.

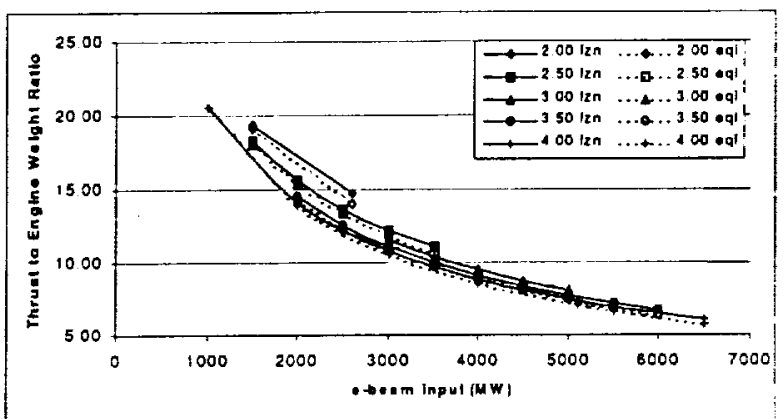

Figure 5. Thrust to engine Weight vs. e-beam energy input

Figure 6 compares the overall efficiency of the engine to energy input for several combustor area ratios. Here the trend between overall efficiency and energy input is downward. The overall efficiency drops significantly at lower energy inputs but approaches a more gradual downward slope at higher energy inputs. For a given energy input the overall efficiency increases with decreasing combustor expansion ratios. Again, the equilibrium values lag those calculated using frozen assumptions.

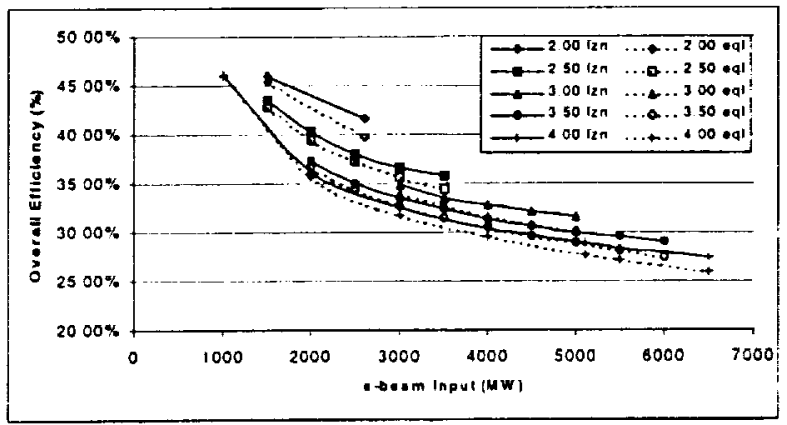

Figure 6. Overall efficiency vs. e-beam energy input.

At this point it is instructive to consider the axial property profiles for the cases above and try to draw some inference on the relationship between the geometry of the profiles and the engine performance. Figure 7 illustrates the change in specific entropy with axial position for all of the cases using the 4.0 combustor area ratio. All of the axial profiles to follow use the equilibrium values only. The zero is impacted on these graphs because we are interested in the change in entropy for that at the combustor inlet, which is the same for all cases. Immediately evident is that the change in entropy progresses more rapidly as the energy input increases. This is intuitive, as heat input to an open system, by which this system can be modeled, will increase entropy. There is also a slight but detectible increase in entropy in the expansion section. There is an entropy increase related to the relaxation of the vibrational and electronic modes in the air molecules.

Figure 7 illustrates the axial entropy profiles for several heat input rates and a combustor expansion ratio of 4.0. For purposes of clarity all of the axial property profiles use the equilibrium data only. The entropy rapidly increases in the combustor section. The lower energy input rates show a near linear increase and the higher energy inputs demonstrate a convex curving shape. The entropy increase is near constant in the expansion section. The slight increase in entropy can be accounted for by the relaxation of excited vibrational and electronic modes in the air molecules. The considerable change in entropy between the lower and higher energy rates accounts for the loss of overall efficiency as demonstrated in Figure 6.

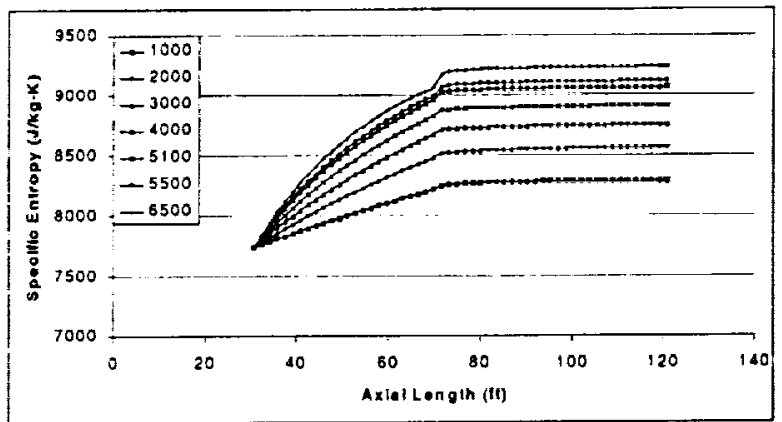

Figure 7. Specific Entropy axial profile for e-beam input and combustor expansion of 4.0 .

Figure 8 is the comparable Mach profiles for Figure 7. Again only the equilibrium values are shown here. The airflows corresponding to the lower energy input rates manage to accelerate through the combustor as well as the expansion sections. As the energy input increases the flow is driven closer to a thermal choke condition. Due to the limitations of the code and from a desire to leave a margin of safety none of the flows are decelerated below a Mach number of 1.2. The higher energy inputs have a higher entropy profile and have been driven closer to a choked condition, all of which is expected from basic compressible relations. Although expected these relations help to explain the efficiency changes between the low and high energy input rates.

Figure 9 plots the entropy profiles for four different combustor area ratios using an e-beam energy input of 
$3000 \mathrm{MW}$. The near linear entropy profiles persist in this configuration. The slight increase in entropy in the expansion section is also evident. The entropy in the combustor section tends to a lower rate of increase with the lower combustor expansion ratios. Also notable is the rate of change of entropy does not seem to be affected in the expansion area for different expansion ratios. The entropy profiles are in agreement with the trends shown in the overall efficiency profiles. The lower entropy cases correspond with the higher overall efficiencies.

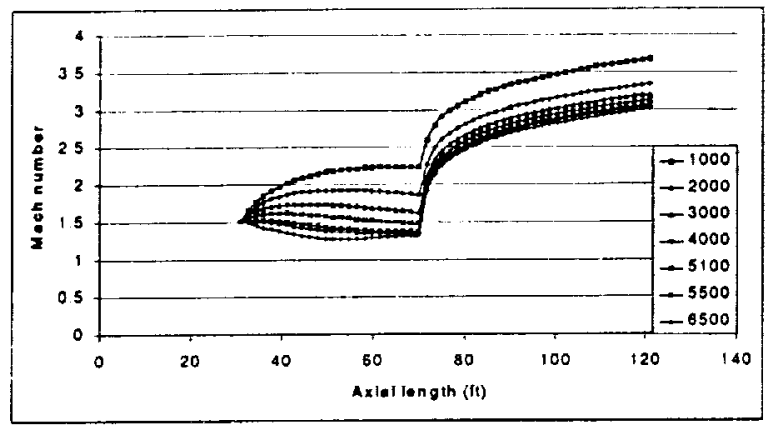

Figure 8. Mach number axial profile for e-beam input and combustor expansion of 4.0 .

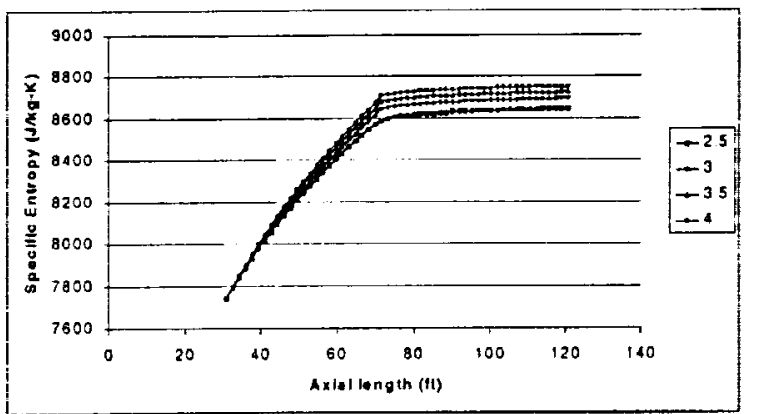

Figure 9. Axial entropy profiles for $3000 \mathrm{MW}$ e-beam input.

Figure 10 is the comparable Mach number plot to Figure 8. Immediately evident is the choking effect experienced by the lower area ratios. The fact that the lower expansion profiles experience a lower entropy change is counterintuitive. One would have expected the flow that more closely approaches a choked condition to have the higher entropy increase.

The change in Mach number at the combustorexpansion interface is more pronounced than in Figure 10 due to the higher expansion area ratios. The change in expansion area ratio did not seem to have a significant effect on the entropy profiles.

Table 3 lists the cases analyzed using the HF laser subsystem. The table is similar to that of Table 1. Here again the intent is to compare the effects of variance of energy input and combustor area ratio on the performance of the SHX engine. The range of energy inputs is smaller for the HF subsystem. The HF laser analysis assumes that the beams are fired at the interface between the combustor and expansion sections. The beams are directed axially upstream in the combustor flowpath. Thus the majority of the beam energy is absorbed near the exit to the combustor. This high local heating rate forces a thermal choke more quickly than if the energy was uniformly absorbed. For similar reasons the range of acceptable combustor area ratios is limited. The 2.0 ratio case is not represented here, as it was not possible to absorb a reasonable level of beam energy without choking.

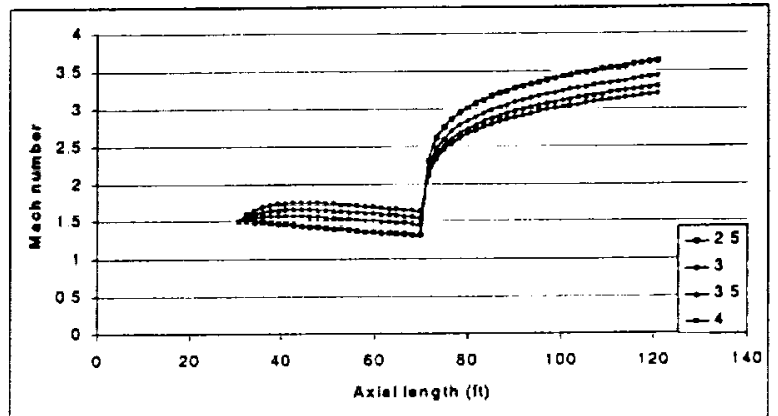

Figure 10. Mach number profiles for $3000 \mathrm{MW}$ ebeam input.

Table 3. Cases analyzed using the HF laser subsystem

\begin{tabular}{|c|c|c|c|}
\hline \multirow[b]{2}{*}{ Case } & \multicolumn{2}{|c|}{ Area Ratio } & \multirow{2}{*}{$\begin{array}{l}\text { Heat } \\
\text { Input }\end{array}$} \\
\hline & Combustor & Expansion & \\
\hline 1 & 2.5 & 7.2 & 2000 \\
\hline 2 & 2.5 & 7.2 & 2500 \\
\hline 3 & 2.5 & 7.2 & 3000 \\
\hline 4 & 3.0 & 6.0 & 1000 \\
\hline 5 & 3.0 & 6.0 & 1500 \\
\hline 6 & 3.0 & 6.0 & 2000 \\
\hline 7 & 3.0 & 6.0 & 2500 \\
\hline 8 & 3.0 & 6.0 & 3100 \\
\hline 9 & 3.5 & 5.14 & 2000 \\
\hline 10 & 3.5 & 5.14 & 2500 \\
\hline 11 & 3.5 & 5.14 & 3000 \\
\hline 12 & 3.5 & 5.14 & 3500 \\
\hline 13 & 4.0 & 4.5 & 2000 \\
\hline 14 & 4.0 & 4.5 & 2500 \\
\hline 15 & 4.0 & 4.5 & 3000 \\
\hline 16 & 4.0 & 4.5 & 3500 \\
\hline 17 & 4.0 & 4.5 & 4000 \\
\hline
\end{tabular}

Figure 11 illustrates the variance in specific thrust with energy input for several combustor area ratios. There are several similarities with Figure 4. The trends are roughly linear and increasing with heat input. For a given energy input specific thrust trends downward with increasing combustor area ratio. Finally the equilibrium values lag those calculated under frozen assumptions. There are also some interesting variations with the ebeam case. First the values reported here significantly 
lag those reported in the e-beam case. Careful comparison of Figure 11 and Figure 4 reveals that the difference between specific thrusts increases with energy input. The reported values for specific thrust is low enough to suggest the HF laser case as designed would not be competitive with a conventional scramjet. Also notable is the nonlinear relationship between energy input and specific thrust at the higher energy levels. One explanation is that since a higher percentage of the total energy is input at the aft part of the combustor, increasing energy input gives diminishing returns in terms of thrust.

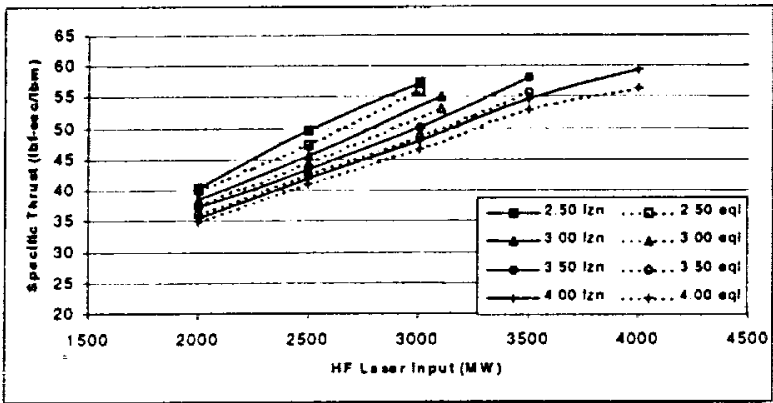

Figure 11. Specific Thrust vs. HF laser power input

Figure 12 plots the thrust to engine weight ratio vs. energy input. The trends of lower thrust to weight ratios with higher energy input and higher combustor area ratios are consistent with the e-beam analysis. The trends seem to maintain a relatively linear profile, which is not consistent with the e-beam case. The diminishing returns on heat input exhibited for specific thrust seems to have 'straightened out' the thrust to engine weight ratio trends. Also notable is the range of the reported thrust to weight ratios. The values shown for the HF laser are significantly lower than to that of the e-beam case. These values are not competitive with a conventional scramjet. The ratios are lower due to the requirement of a $\mathrm{HF}$ cycling mass, discussed in the previous section, to convert the electrical energy from the fusion reactor to chemical energy needed in the laser.

The overall efficiency plots, shown in Figure 13, illustrate a similar linearly decreasing trend with energy input. The values reported here also illustrate a reduction in efficiency from the e-beam case. In all other respects the trends in Figure 13 are consistent with those reported by the e-beam analysis.

Figure 14 shows the axial entropy profiles for all of the cases using the 4.0 combustor expansion ratio. Evident is the concave curves in the combustor region, consistent with an increase in the rate of change of entropy. This phenomenon is explained by the higher heating rates experienced in the aft part of the combustor for this configuration. However the exit entropy is not significantly different to the cases with corresponding energy input rates for the e-beam case. This suggests that the difference in specific thrusts is due to a lack of adequate expansion and not from a loss of available work.

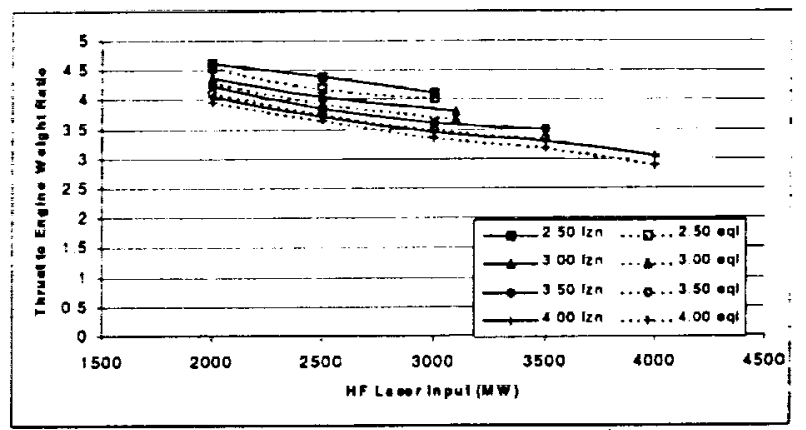

Figure 12. Thrust to engine weight vs. HF laser power input

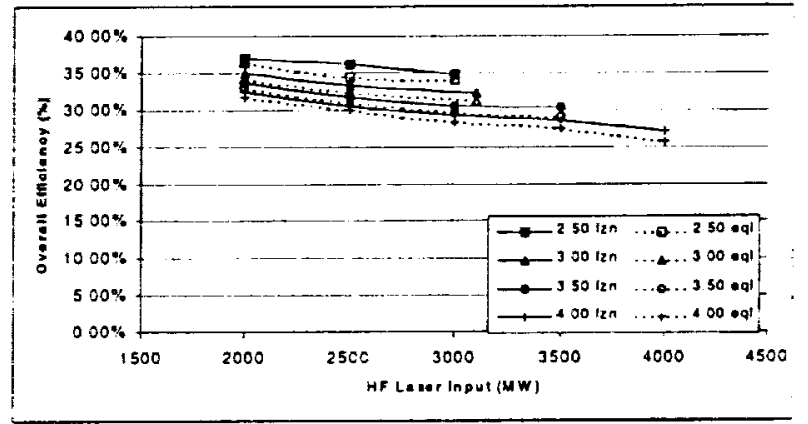

Figure 13. Overall efficiency vs. HF laser power input.

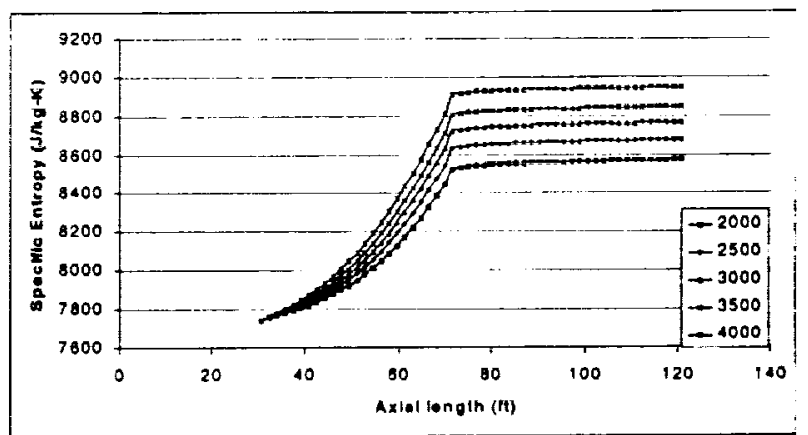

Figure 14. Axial specific entropy profile for 4.0 combustor area ratio and HF laser mode.

Figure 15 illustrates the corresponding axial Mach profile to that of Figure 14. The high heating rates in the aft section of the combustor are again evident by the strong deceleration of the flow in the latter half of the combustor. As expected, the deceleration is stronger for the higher heating rates.

Figure 16 shows the specific entropy axial profiles using an HF laser energy input of $2500 \mathrm{MW}$. Again the combustor profiles are concave where the e-beam profiles are convex. However the higher expansion 
ratios are experiencing the higher changes in entropy, which is consistent with the results in the e-beam analysis. Figure 17 illustrates the corresponding Mach number profiles. Again the lower combustor area ratios are driven closest to a thermal choke and experience the greatest acceleration in the expansion section.

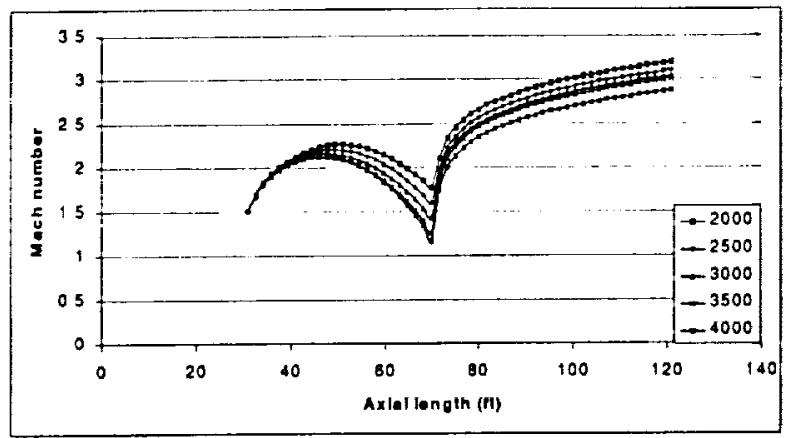

Figure 15. Axial Mach number profile for 4.0 combustor area ratio and HF laser mode

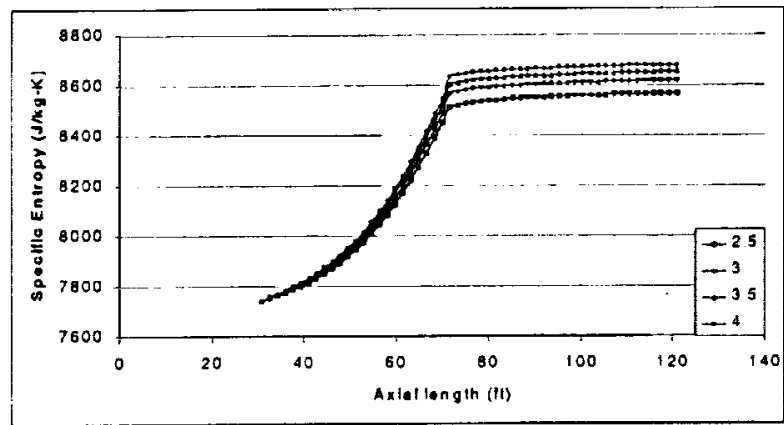

Figure 16. Axial specific entropy profile for HF laser power input of $2500 \mathrm{MW}$.

Several cases were run using the e-beam and $\mathrm{HF}$ laser subsystems to couple the power output from the fusion reactor to the engine flowpath. Both subsystems exhibited similar trends. Specific thrust increased with power input and decreased with higher combustor area ratios. Thrust to weight ratio and overall efficiency decreased with power input and combustor area ratio. In all cases the equilibrium performance values lagged the frozen values. The results suggest that a trade off will be required between specific thrust and thrust to weight ratio each specific vehicle and mission considered. The power input for the vehicle will be driven by the required specific thrust. Also the flow should be driven to as near a thermal choke as possible by selecting the minimum combustor area profile that will handle the input power.

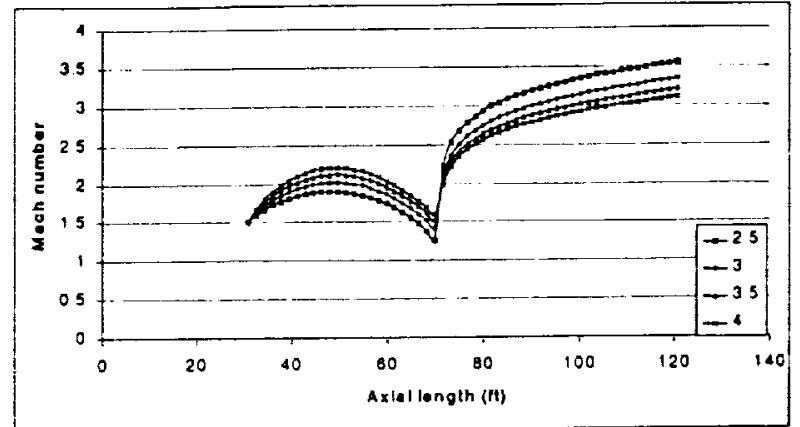

Figure 17. Axial Mach number profile for HF laser power input of $2500 \mathrm{MW}$

\section{CONCLUSIONS}

The HF laser showed significantly lower performance than the e-beam mode for all three metrics due to the non-uniform heating profile, incomplete absorption and necessity for additional $\mathrm{HF}$ reactant mass. If the HF laser subsystem must be evaluated over the e-beam subsystem due to other factors it would be advisable to consider other laser configurations that the aft combustor firing forward used in this model.

Specific entropy and Mach profiles were generated for both subsystems. Trends were as expected for all cases and partially explained the variance in performance for the individual cases.

\section{ACKNOWLEDGEMENTS}

Creation of the initial SHX code was funded in part through a NASA Phase I Small Business Innovative Research contract awarded to International Space Systems, Inc.

1 Heiser, W. H. and Pratt, D. T., Hypersonic Airbreathing Propulsion, American Institute of Aeronautics and Astronautics, Inc., Washington, DC, 1994.

${ }^{2}$ Bussard, R. W., "The QED Engine System, Direct Electric Fusion Powered Rocket Propulsion Systems", $10^{\text {hh }}$ Symposium on Space Nuclear Power and Propulsion, American Institute of Physics, New York, NY, 1993.

3 Adams, R. B., "Preliminary Analysis of a FusionElectric Transatmospheric Airbreathing Vehicle", M.S. Thesis, University of Alabama in Huntsville, 2000.

${ }^{4}$ Adams, R. B., Landrum, D. B., "Preliminary Analysis of an Earth to Orbit Fusion Powered Airbreathing Vehicle", AIAA Paper 2000-3366, June 2000. 